\title{
Design of the Fingerprint Machine Circuit for the Intelligent Managerial System of Blasting Equipment Library
}

\author{
Xingshan $\mathrm{LI}^{1, \text { a }}$, Luhua Zhao ${ }^{2}$ \\ ${ }^{1}$ Luohe medical college, Luohe, 462000, China; \\ ${ }^{2}$ Henan quality engineering vocational college, Pingdingshan,475000, China \\ aemail:604141388@qq.com
}

Keywords: Coal mine blasting equipment library; Intelligence; single chip microcomputer (SCM); Wiegand protocol; Fingerprint machine

\begin{abstract}
This paper mainly analyses the necessity to manage the explosive in the coal mine blasting equipment library. It make intelligent management possible by using digital monitoring technology, communication technology and software technology and sensor technology for reference; using high performance microcontroller to identify if the fingerprint is legitimate; then control the opening and closing of the electronic lock.
\end{abstract}

\section{Introduction}

In order to ensure the safety in the production of coal mine,the storage of powder and its access management must be strict and intelligent.With the rapid development of digital control technology, we can make intelligent management possible by using the electronic lock and the fingerprint machine.

\section{Component of coal mining blasting equipment library system}

The intelligent management system is made up of three parts: the lower machine, the control device and PC. The system is shown as Fig.1.

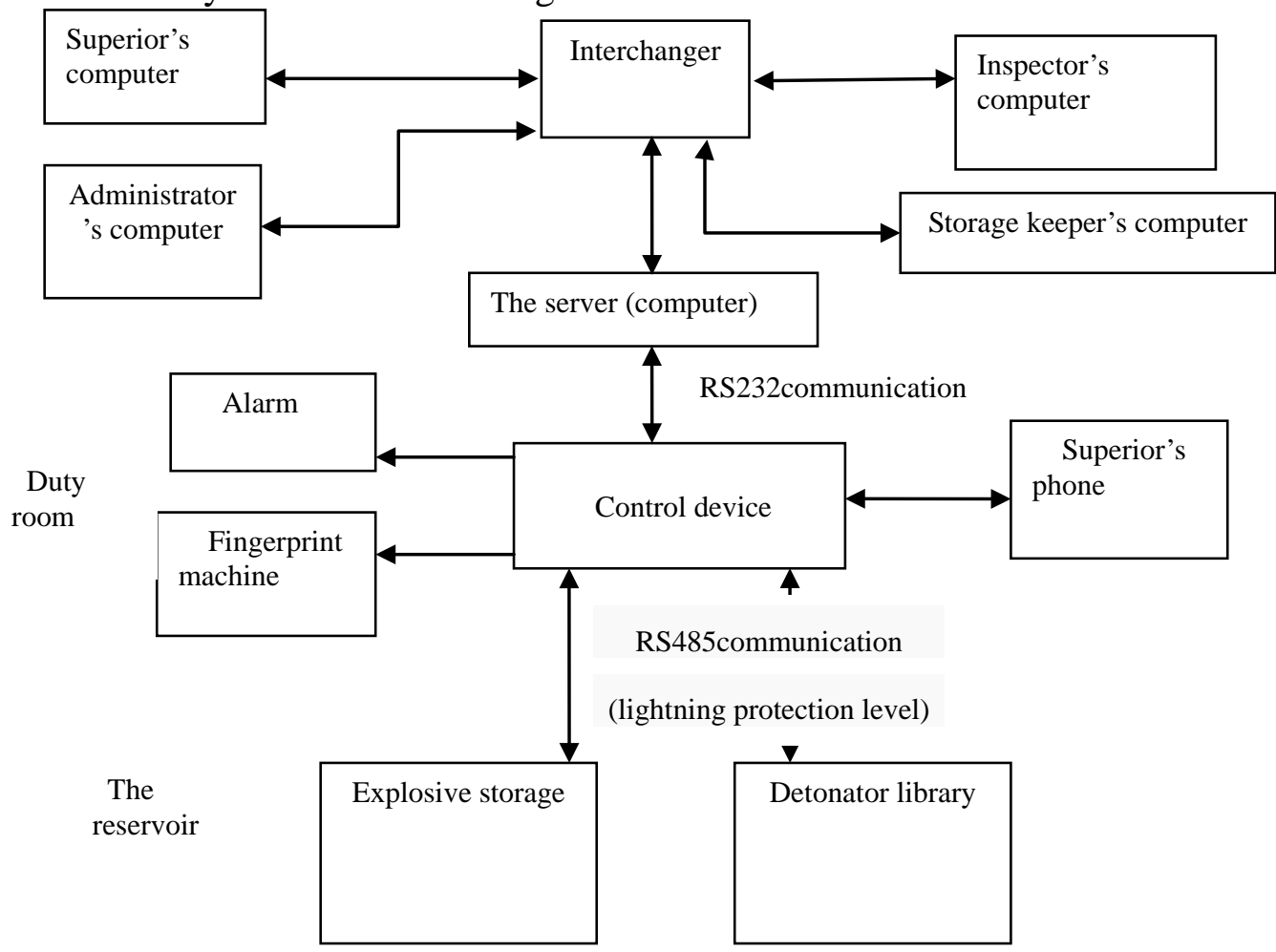

Fig.1. Managerial system of the coal mining blasting equipment library 


\section{Control system of the lower machine}

The storage and access of the explosives and detonators in the reservoir area are managed by the lower machine (computer entrance guard system). The control system of lower machine is mainly composed of single chip microcomputer STC12C5A60S, temperature and humidity sensor, vibration sensor, the fingerprint machine, electromagnetic lock control and communication module. The lower machine uses single chip to control the system [1]. The system is shown as Fig.2.

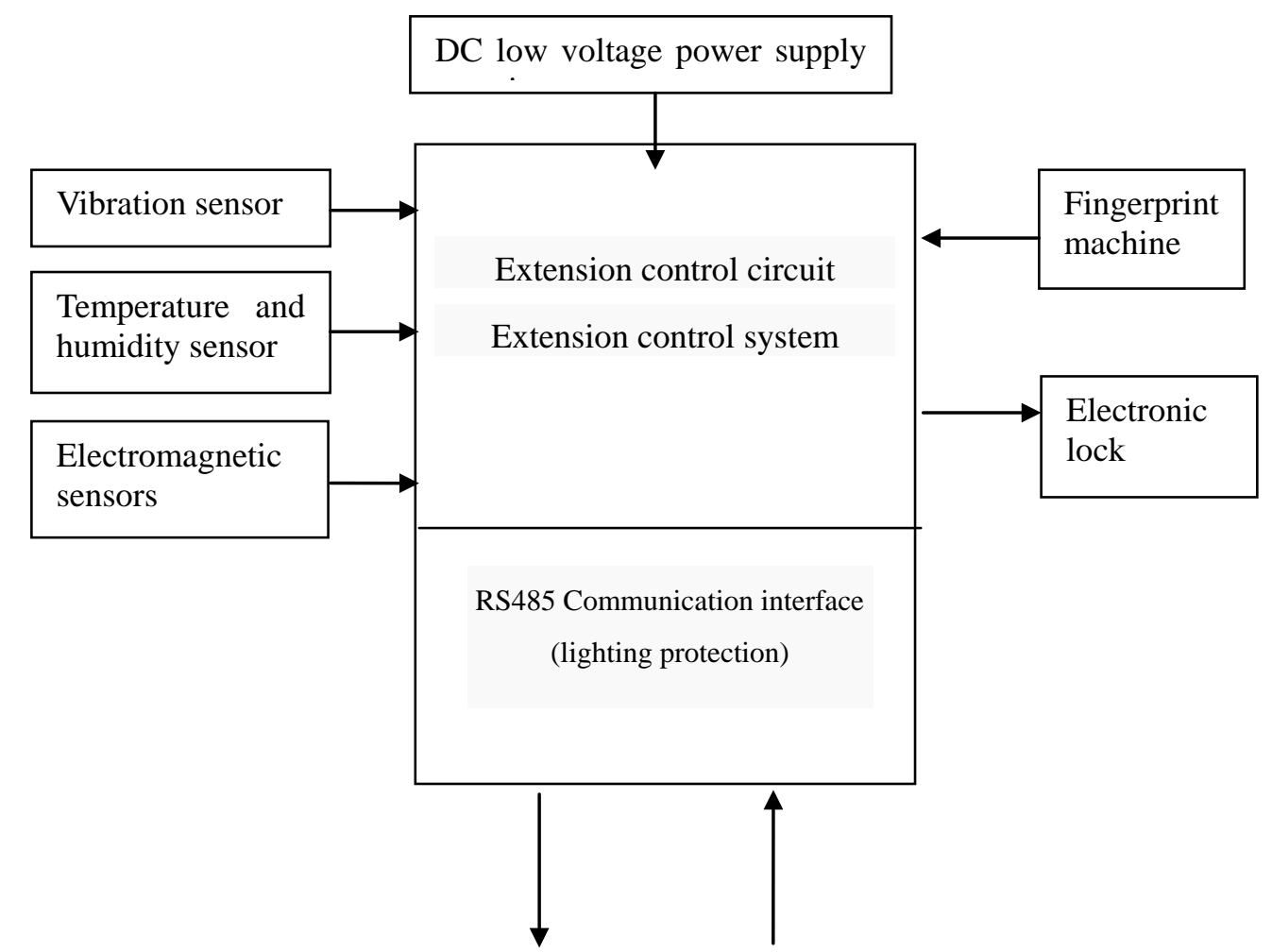

Fig.2. Control system of lower machine for coal mining blasting equipment library

\section{The acquisition of fingerprint and implementation of Wiegand interface circuit}

The blasting equipment library uses the electronic lock (spiritual lock) to open and close the door. The intelligent spiritual is a new product, it is based on the working principle of electronic control door, it also combined the advantages of the electronic control door and the magnetic door [2].

(1)The acquisition of fingerprint information

First, we need to register the personnel's fingerprint on the fingerprint machine, and then send these fingerprint information to single chip microcomputer and upload the information to PC. PC will register the specific fingerprint information. While using this system, the machine will compare the different fingerprints of warehouse keeper and the man who want to get the explosives, if their fingerprints can be matched, they can get the permission to get the explosives.

(2)Leaders use management system or mobile phone license to open the door

When the fingerprints of the warehouse keeper and the man who wants to get explosives are matched correctly, the lower machine will send a request to the managerial system to open the door, if the superior is besides the computer, he can give the permission to open the door by clicking the button in the PC managerial system. After he gives the authorization, the man who wants to get the explosives can press his fingerprint, then the electronic, which is controlled by single chip microcomputer will open automatically. If the superior is not besides the computer, the system will call the superior, and the superior will send the authorization code to open the door.

(3)Fingerprint identification

To open the door of the blasting equipment library, we must be certified. One of the most important steps is to identify the fingerprint of the registered personnel, so the lower machine 
system must have the function to identify the fingerprints. Every person's fingerprints contains a large amount of information, the lines of fingerprints has different images, breakpoints and intersections. Depends on the unique characteristics of fingerprints, we can match a man to his fingerprints correctly. By comparing the features of fingerprints and the preserved fingerprints, we can verify his real identity. We can verify a person's identity by using photoelectric conversion device and computer image processing technology and collecting, analyzing and comparing living's fingerprints [3]. The system uses ZDC1535 fingerprint machine. This machine can register and store the information of about 60000 different fingerprints. It has the function to output the data of fingerprints, which can be easily connected with the single chip microcomputer. It used the widely-used international Wiegand protocol, which has the function of secondary development.

(4) Wiegand protocol

In terms of fingerprint identification, it uses the fingerprint machine which has the output of Wiegand protocol, its main control chip can read the information of fingerprint and process the data. The main technical point of reading and operating the fingerprint information is that the system uses STC12C5A60S2 based on Wiegand protocol to read the information. Wiegand protocol is a way of communication developed byMOTOROLA,it is mainly used in the products of fingerprints machine, entrance guard and other products. Its protocol is free, which does not define the transfer rate or the length of data transmission. Its core is the way of data transmission.

The present products with Wiegand protocol on the market use 26 or 34 data format [4].

The device outputted based on Wiegand protocol is consist of two wires and a GND thread. The two wires are respectively DATA0 and DATA1, DATA0 represents the transmission of ' 0 ', DATA1 represents the transmission of ' 1 '. So we can find that Wiegand protocol data transmission is a simplex mode transmission. In the absence of data transmission, the two lines DATA0 and DATA1 keep the high level of $+5 \mathrm{v}$. while sending the ' 0 ' signal, line DATA0 sends a low pulse signal. In the same way, it will send the signal ' 1 ' while the line DATA1sends a low pulse signal [5] [6]. The transmission characteristics can use 51 single-chip microcomputer to capture two external interrupt line, which will make it easier to implement the code.

\section{Circuit design}

The design SCM and electronic lock circuit interface, which uses 4 pin and the logic circuit made up of two 74LS00 chips to drive the work of the electronic lock, its purpose is to prevent the interference or equipment malfunctions. As it is related to the success of the project if the lock is open correctly. Obviously, this design is more reliable and safer. The electronic lock is a DC motor $12 \mathrm{~V}$, it is enough to use optocoupler isolation Jada Layton B772 to drive the action.The communication interface between the single chip microcomputer and the fingerprint machine can be connected directly by the two external interrupt pin of the single chip microcomputer and the two lines DATA0, DATA1 of the fingerprint machine based on Wiegand protocol. It can read the data ' 0 ' and data ' 1 ' in the interrupted program. To increase the anti-interference and the transmission distance, we can pull up 10k resistor in each side of the single chip microcomputer lines.

The interface circuit of the lower machine and fingerprint machine is shown as Fig.3: 

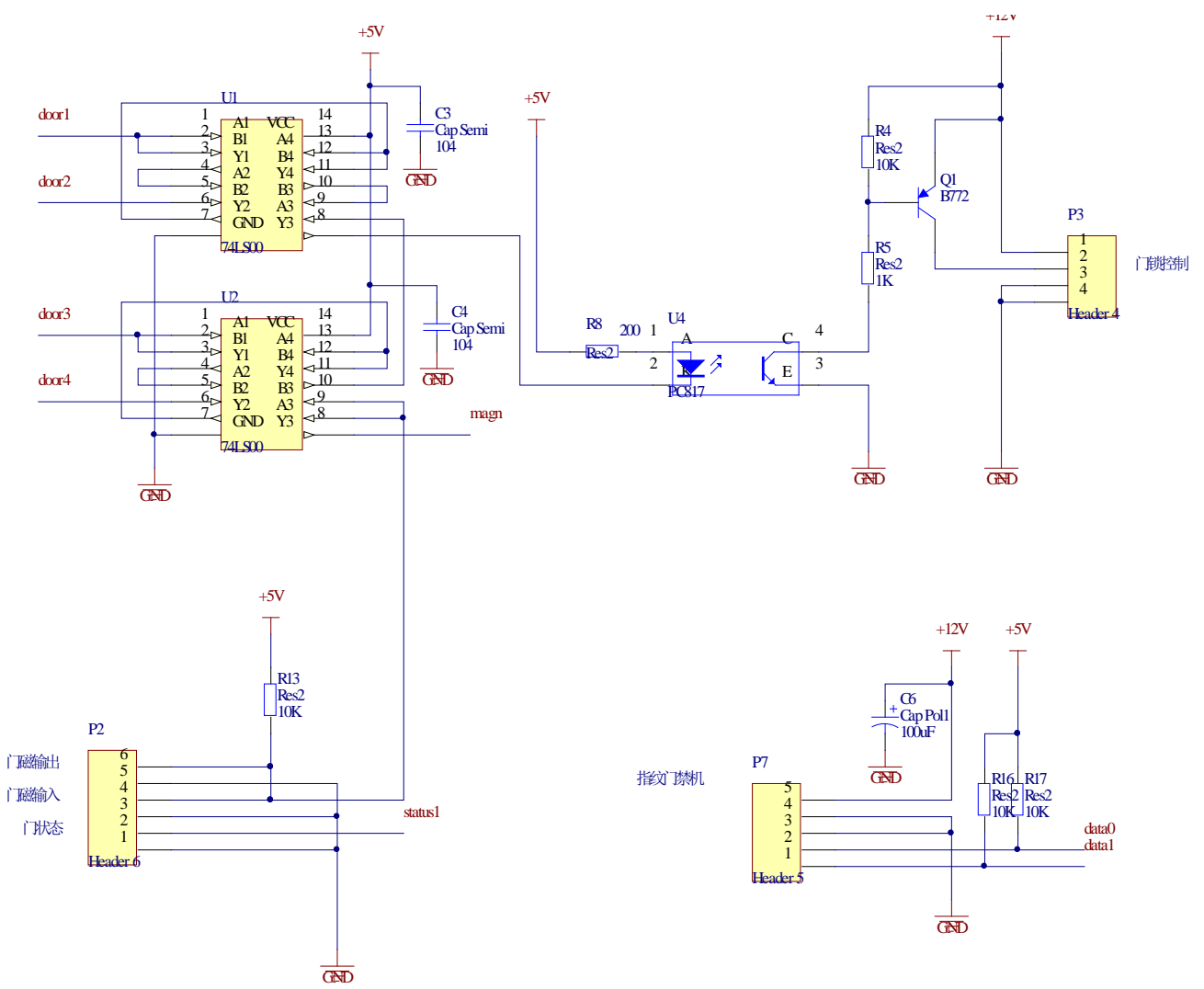

Fig.3. Electronic lock control with the fingerprint machine interface circuit

The main control chip of the lower machine STC12C5A60S2 and its electronic lock drive are based on Wiegand protocol. The DATA0 fingerprint machine adopts $\mathrm{C}$ functions.

\section{Authorization module to open the door}

The system requires that the door can only be open under the condition of the warehouse keeper coordinates with a pickup or delivery. So when the fingerprint of open the door request arrives, the hardware will identify and judge the fingerprint, if the conditions are matched, it will send a request to the management software to open the door; otherwise it will be reset to the initial state. After the management software receives two request to open the door when the conditions are met, the dialog box pop-up prompts the user to grant or deny. The design process is shown in Fig.4.

\section{Conclusion}

Through reasonable design and a lot of experiments of fingerprint machine circuit, the experiment parameters of coal mining blasting equipment library can reach the expected indicators, avoid safety accidents caused by explosives from the source. This system achieves the intelligent management for the storage and access for explosives and detonators, which can avoid the safety accidents from the greatest degree of caused by such sources.

\section{Acknowledgement}

In this paper, the research was sponsored by the Nature Science Foundation of Henan Province (Project No. 142300410286) and by the Nature Science Foundation of Luohe medical college(2013-S-LMC27).

\section{Reference}

[1] Chenwei Cai, Haijian, Fan, Biyang Liu.Design and Application of Gate Control System Based on Fingerprint Identification Technology[J],Video Engineering. 2012.36(3) 128-130 
[2] Xunyu Zhong, Bingqi Wang, Bing Li. Design of apparatus-cabinet controller based on EPM7128SLC84[J].Journal Of Modern Electronics Technique.2006(1) 110-112

[3] Yingchun Zhong, Guochen Huang. Electronic Equipment Isolation Management System withFingerprint Recognition[J].MICROPROCESSORS.2012(4) 76-79

[4] Yunfeng Yue, Rui Wang, Haitao Sun and so on. Application of Wiegand protocol[J]. Journal Of Qiqihar University（Natural Science Edition.2002,18(2) 56-58.

[5] Deheng Xu. Multi-channel Wiegand signal acquiring and processing based on SCM[J].Computer Measurement \& Control, 2004,12(4)375-377

[6] Jiong Zhang. Multi-channel wiegand signal acquiring and processing based on ARM7[J].Microcontrollers \& Embedded Systems. 2008(2)67-69

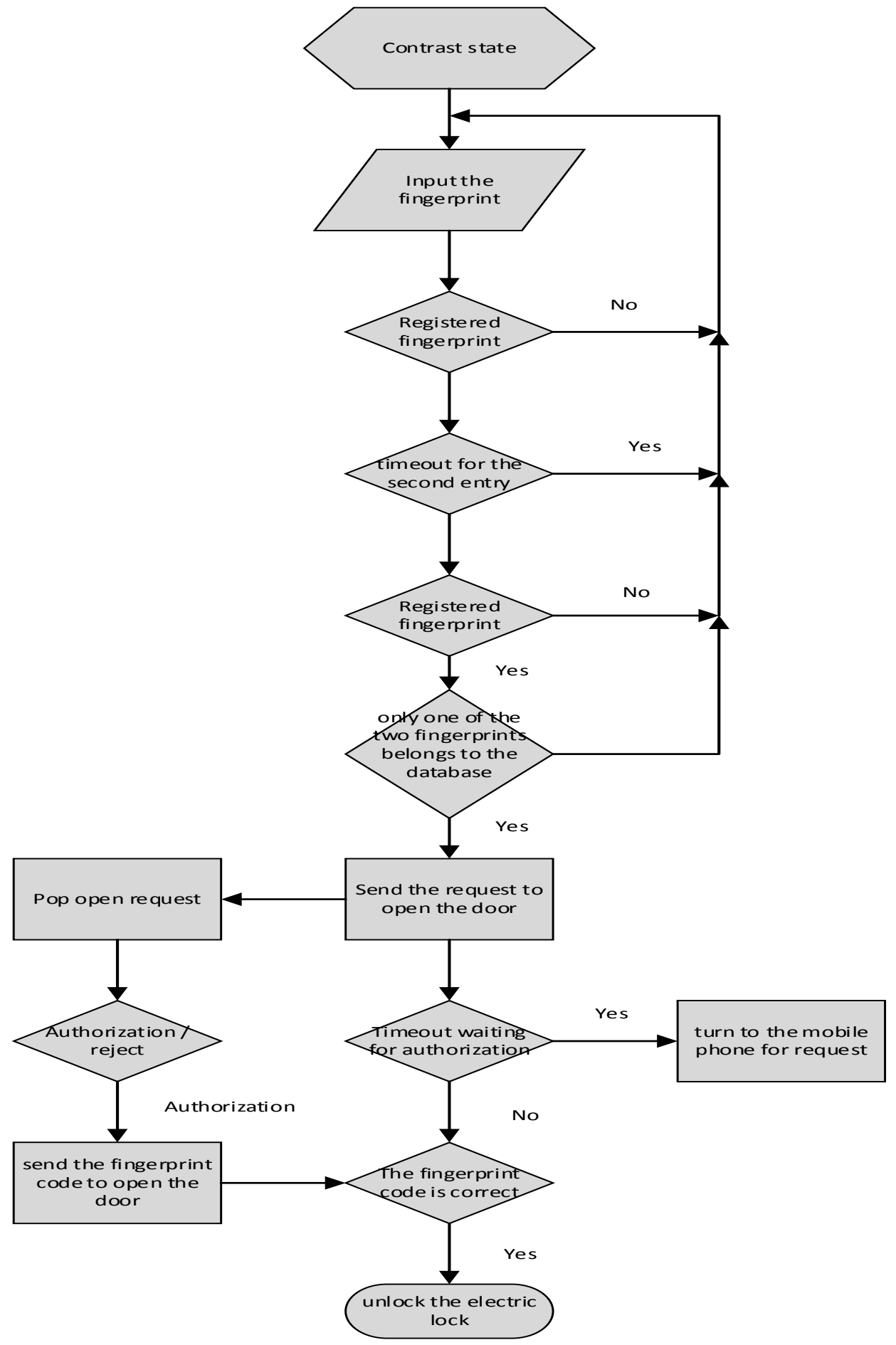

Fig.4. flow chart to open the door 